\title{
Horizon Thermodynamics and Gravitational Field Equations in Quasi-Topological Gravity
}

\author{
A. Sheykhi ${ }^{1,2}$, M. H. Dehghani ${ }^{1,2}$ and R. Dehghani ${ }^{1}$ \\ 1 Physics Department and Biruni Observatory, College of Sciences, Shiraz University, Shiraz 71454, Iran \\ ${ }^{2}$ Research Institute for Astronomy and Astrophysics of Maragha (RIAAM), P.O. Box 55134-441, Maragha, Iran
}

\begin{abstract}
In this paper we show that the gravitational field equations of $(n+1)$-dimensional topological black holes with constant horizon curvature, in cubic and quartic quasi-topological gravity, can be recast in the form of the first law of thermodynamics, $d E=T d S-P d V$, at the black hole horizon. This procedure leads to extract an expression for the horizon entropy as well as the energy (mass) in terms of the horizon radius, which coincide exactly with those obtained in quasi-topological gravity by solving the field equations and using the Wald's method. We also argue that this approach is powerful and can be extended to all higher order quasi-topological gravity for extracting the corresponding entropy and energy in terms of horizon radius.
\end{abstract}

Keywords: quasi-topological; thermodynamics; gravity. reconstruction.

\section{INTRODUCTION}

In recent years, most theoretical physicists as well as cosmologists, have been convinced that there should be a deep relation between the gravitational field equations and the laws of thermodynamics. It was pointed out by Jacobson for the first time in 1995, that the hyperbolic second order partial differential Einstein equation has a predisposition to the first law of thermodynamics [1]. Indeed, Jacobson derived the Einstein field equations of general relativity, in its tensorial form, by applying the Clausius relation, $\delta Q=T \delta S$, on the horizon of spacetime, here $\delta S$ is the change in the entropy and $\delta Q$ and $T$ are the energy flux across the horizon and the Unruh temperature seen by an accelerating observer just inside the horizon. Also, by applying the Clauius relation to the apparent horizon of the FriedmannRobertson-Walker universe, the corresponding Friedmann equations can be derived in Einstein, Gauss-Bonnet and more general Lovelock gravity 2]. Following these investigations, a lot of attempts have been done to reveal the connection between thermodynamics and gravity in different setups [3-12]. For example, in Ref. [13] the relationship between the first law of thermodynamics and the gravitational field equation of a static, spherically symmetric black hole in Horava-Lifshitz gravity has been explored. It was shown that, the gravitational field equations of static, spherically symmetric black holes in Horava-Lifshitz theory can be expressed as the first law of thermodynamics on the event horizon [13]. This approach can lead to extract expressions for the entropy and mass of Horava-Lifshitz black holes which are consistent with those obtained from other approaches [13]. These results further support the idea that gravitation on a macroscopic scale is a manifestation of thermodynamics.

It is well known that the natural generalization of the Einstein-Hilbert action to higher dimensional spacetime, and higher order gravity with second order equation of motion, is the Lovelock action [14]. However, because of the topological origin of the Lovelock terms, the second term of the Lovelock action (the Gauss-Bonnet term) does not have any dynamical effect in four dimensions. Similarly, the cubic interaction only contributes to the equations of motion when the bulk dimension is seven or greater. Recently, a modification of third order Lovelock gravity was proposed by [15, 16] which contains cubic terms of Riemann tensor and contribute to the equation of motions in five dimensions. This new theory, which is called "quasi-topological" gravity, was also extended to include the quartic terms of Riemann tensor [17]. Quasi-topological gravity provides a useful toy model to study a broader class of four (and higher) dimensional CFT's, involving three or more independent parameters [18]. Black hole spacetimes in higher order quasi-topological gravity which have at most second order derivatives of the metric in the field equations, have been explored and their thermodynamics have been investigated 1922 .

In this paper we turn to investigate the connection between the gravitational field equations and the first law of thermodynamics in quasi-topological gravity. For a static topological black hole spacetime with constant horizon curvature, we will show that the gravitational field equations can be transformed to the first law of thermodynamics, $T d S-d E=P d V$, on the black hole horizon. This allows us to extract the entropy expression in terms of the horizon radius, which is useful in studying thermodynamics of these kind of black holes.

The structure of this paper is as follow. In the next section, we show that the gravitational field equations in Einstein and Gauss-Bonnet gravity can be recast as the first law of thermodynamics on the black hole horizon. In section III, we briefly review the action of the quasi-topological gravity. In section IV, we apply the method to cubic quasi-topological theory. In section $\mathrm{V}$, we will generalize our approach to the quartic and higher order quasi-topological gravity and extract the entropy and energy expressions of these theories. We finish our paper with conclusions in section VI. 


\section{HORIZON THERMODYNAMICS IN EINSTEIN AND GAUSS-BONNET GRAVITY}

Let us start with Einstein-Hilbert and Gauss-Bonnet cases to set the stage and to see how the method works [3]. One can derive the equations of motion for gravitational theory by either, varying the action of the theory with respect to $g_{\mu \nu}$, without specifying the form of the spacetime metric, or by specifying the spacetime metric, then inserting the metric in the action, and finally varying the resulting action with respect to the metric functions. In both cases one arrives at the field equations. To see how the two approaches leads to the same result, in this section, we use both the field equations as well as variation method, for transforming the equations of motion to the first law of thermodynamics at the black hole horizon.

\section{A. The Einstein-Hilbert Case}

The Einstein field equations is $(c=1)$

$$
R_{a b}-\frac{1}{2} R g_{a b}=8 \pi G T_{a b}
$$

We consider a four-dimensional static, spherically symmetric spacetime with a horizon, which is described by the metric

$$
d s^{2}=-f(r) d t^{2}+\frac{d r^{2}}{f(r)}+r^{2} d \Omega^{2} .
$$

Inserting metric (2) in Eq. (1), the $(r r)$ component of the Einstein equations can be written

$$
\frac{1}{r^{2}}\left[r f^{\prime}(r)-1+f(r)\right]=8 \pi G P,
$$

where $P=T_{r}^{r}$ is the radial pressure of matter at the horizon [13]. Here prime denotes derivative with respect to $r$. We assume that the spacetime has a horizon at $r=r_{+}$which is the simple root of $f\left(r_{+}\right)=0$. We also propose at $r=r_{+}$ the surface gravity $\kappa=f^{\prime}\left(r_{+}\right) / 2$ has non zero value which implies a finite non zero temperature $T=f^{\prime}\left(r_{+}\right) / 4 \pi$ at the horizon. Evaluating the field equation (3) at the horizon where $f\left(r_{+}\right)=0$, we find

$$
\frac{1}{G}\left(\frac{r_{+} f^{\prime}\left(r_{+}\right)}{2}-\frac{1}{2}\right)=4 \pi r_{+}^{2} P .
$$

Multiplying both sides of Eq. (4) by $d r_{+}$, we arrive at

$$
\underbrace{\frac{f^{\prime}\left(r_{+}\right)}{4 \pi}}_{\mathrm{T}} \underbrace{\frac{1}{G} d\left(\frac{4 \pi r_{+}^{2}}{4}\right)}_{\mathrm{dS}} \underbrace{-\frac{1}{2}\left(\frac{d r_{+}}{G}\right)}_{\text {-dE }}=\underbrace{\operatorname{Pd}\left(\frac{4 \pi r_{+}^{3}}{3}\right)}_{\mathrm{P} \mathrm{d} \mathrm{V}} .
$$

If we invoke the expressions for the entropy and energy (mass) of the black hole,

$$
\begin{aligned}
& S=\frac{A}{4 G} \\
& E=\frac{r_{+}}{2 G}
\end{aligned}
$$

where $A=4 \pi r_{+}^{2}$ is the area of the horizon, we find that Eq. (5) is nothing but the first law of thermodynamics,

$$
d E=T d S-P d V
$$

\section{B. The Gauss-Bonnet Gravity}

We shall now turn our attention to the more general case, namely the Gauss-Bonnet gravity. In an effective action approach to the string theory, the Gauss-Bonnet term corresponds to the leading order quantum corrections to gravity, and its presence guarantees a ghost-free action [23]. This theory contains a special combination of curvature-squared 
term, added to the Einstein-Hilbert action. The Gauss-Bonnet term does not have any dynamical effect in four dimensions since it is just a topological term in four dimensions. Static black hole solutions of Gauss-Bonnet gravity have been found and their thermodynamics have been investigated in ample details [24]. The action of the EinsteinHilbert in the presence of the Gauss-Bonnet correction term, in $(n+1)$-dimensions, is given by

$$
I=\frac{1}{16 \pi G_{n+1}} \int d^{n+1} x \sqrt{-g}\left(-2 \Lambda+R+\alpha \mathcal{L}_{G B}\right)+\int d^{n+1} x \mathcal{L}_{M},
$$

where $\alpha$ is the Gauss-Bonnet coefficient with dimension (length) ${ }^{2}$, and $\mathcal{L}_{G B}$ is the Gauss-Bonnet Lagrangian which has the form,

$$
\mathcal{L}_{G B}=R_{a b c d} R^{a b c d}-4 R_{a b} R^{a b}+R^{2}
$$

The field equations can be obtained by varying the above action with respect to the metric $g_{a b}$. We find

$$
G_{a b}+\Lambda g_{a b}+2 \alpha H_{a b}=8 \pi G_{n+1} T_{a b}
$$

where,

$$
\begin{aligned}
& G_{a b}=R_{a b}-\frac{1}{2} R g_{a b}, \\
& H_{a b}=R R_{a b}-2 R_{a}{ }^{c} R_{b c}-2 R^{c d} R_{a c b d}+R_{a}{ }^{c d e} R_{b c d e}-\frac{1}{4} g_{a b} \mathcal{L}_{G B},
\end{aligned}
$$

are the Einstein and the second-order Lovelock tensor, respectively. Consider again a static spherically symmetric solution of the form

$$
d s^{2}=-f(r) d t^{2}+\frac{d r^{2}}{f(r)}+r^{2} d \Omega_{n-1}^{2}
$$

Substituting metric (14) in Eq. (11), the $(r r)$ component of the field equations reduces to

$$
\frac{1}{2} \frac{(n-1)}{r^{2}}\left\{r f^{\prime}-(n-2)(1-f)+\frac{\tilde{\alpha}}{r^{2}}(1-f)\left[2 r f^{\prime}-(n-4)(1-f)\right]-\frac{n r^{2}}{l^{2}}\right\}=8 \pi G_{n+1} P .
$$

where again $P=T_{r}^{r}$ is the radial pressure of matter at the horizon, and we have defined $\tilde{\alpha}=(n-2)(n-3) \alpha$.

On the other hand, by substituting the metric (14) in action (10), and varying the action (10) with respect to $g^{r r}$, after multiplying both sides in $(-g)^{-1 / 2} g^{r r}$, we get

$$
\frac{(n-1)}{16 \pi G_{n+1}} \frac{1}{r^{2}}\left\{\left(r+\frac{2 \tilde{\alpha}}{r}-\frac{2 \tilde{\alpha} f}{r}\right) f^{\prime}-(n-2)(1-f)-\frac{\tilde{\alpha}}{r^{2}}(n-4)(1-f)^{2}-\frac{n r^{2}}{l^{2}}\right\}=P .
$$

where $P$ is defined as,

$$
P=T_{r}^{r}=g^{r r} T_{r r}=g^{r r}\left\{\frac{2}{\sqrt{-g}} \frac{\delta \mathcal{L}_{M}}{\delta g^{r r}}\right\} .
$$

As one can see, the resulting equation derived by variational principle in (16) is precisely the same as one obtained in (15) directly from the field equations (11). As we mentioned already, we have two approaches for deriving the components of the field equations; varying the action with respect to $g_{\mu \nu}$, and then inserting metric in the field equations, or by substituting the metric in the action, and varying the resulting action with respect to the metric functions.

The variational method leads to (16), can also be applied to the more general metric

$$
d s^{2}=-N^{2}(r) f(r) d t^{2}+\frac{d r^{2}}{f(r)}+r^{2} d \Sigma_{k, n-1}^{2},
$$

where $d \Sigma_{k, n-1}^{2}$ represents the line elements of an $(n-1)$-dimensional constant curvature hypersurface with unit radius. Without loss of the generality, one may take $k=1,-1$, and 0 , corresponding to spherical, hyperbolic and planar hypersurface. Inserting metric (18) in action (10), after variation with respect to $g^{r r}$, we arrive at

$$
\begin{aligned}
& \frac{(n-1)}{16 \pi G_{n+1}} \frac{1}{N r^{3}}\left\{\left(r^{2}+2 k \tilde{\alpha}-2 \tilde{\alpha} f\right)\left(N^{2} f\right)^{\prime}+N^{2}\left[r(n-2)(f-k)-\frac{\tilde{\alpha}}{r}(n-4)\left(k^{2}-2 k f+f^{2}\right)-\frac{n r^{3}}{l^{2}}\right]\right\} \\
& =T_{r}^{r}=P
\end{aligned}
$$


where again the radial pressure is given by (17). We also assume the function $f(r)$ has a simple zero at $r=r_{+}$with $f\left(r=r_{+}\right)=0$ and non-vanishing surface gravity $\kappa=N f^{\prime}\left(r_{+}\right) / 2$. The temperature associated with the horizon is now defined as $T=\kappa / 2 \pi=N f^{\prime}\left(r_{+}\right) / 4 \pi$. Evaluating Eq. (19) at $r=r_{+}$, we obtain

$$
\frac{N(n-1)}{16 \pi G_{n+1} r_{+}^{2}}\left[f^{\prime}\left(r_{+}\right)\left(r_{+}+\tilde{\alpha} \frac{2 k}{r_{+}}\right)-k(n-2)-\frac{\tilde{\alpha}}{r_{+}^{2}} k^{2}(n-4)-\frac{n r_{+}^{2}}{l^{2}}\right]=P .
$$

Consider two equilibrium states of the system with an infinitesimal different in the extensive variables entropy, energy, and volume $d S, d E$ and $d V$, respectively, while the values of the intensive quantities of the system are the temperature $T$ and pressure $P$. Our aim is to introduce in Eq. (20) a factor $d V$ and see whether we can rewrite it in the form $T d S-d E=P d V$. Multiplying both sides of Eq. (20) by the factor $\Sigma_{k} r_{+}^{n-3} d r_{+}$, where $\Sigma_{k}$ is the area of a unit $(n-1)$-dimensional constant hypersurface with volume $V=\Sigma_{k} r_{+}^{n} / n$, we arrive at

$$
\frac{\kappa}{2 \pi} d\left[\frac{\Sigma_{k} r_{+}^{n-1}}{4 G_{n+1}}\left(1+\frac{(n-1)}{(n-3)} \frac{2 k \tilde{\alpha}}{r_{+}^{2}}\right)\right]-d\left[\frac{(n-1) \Sigma_{k} r_{+}^{n-2}}{16 \pi G_{n+1}}\left(k+\frac{\tilde{\alpha} k^{2}}{r_{+}^{2}}+\frac{r_{+}^{2}}{l^{2}}\right)\right]=P \Sigma_{k} r_{+}^{n-1} d r_{+}=P d V .
$$

The first term in the left hand side is in the form $T d S$ and our analysis allows us to read off the expression of entropy $S$ for the horizon as,

$$
S=\frac{\Sigma_{k} r_{+}^{n-1}}{4 G_{n+1}}\left(1+\frac{(n-1)}{(n-3)} \frac{2 \tilde{\alpha} k}{r_{+}^{2}}\right)
$$

In addition, the second term in (21) can be interpreted as $d E$, where the energy of the system is given by

$$
E=\frac{(n-1) \Sigma_{k} r_{+}^{n-2}}{16 \pi G_{n+1}}\left(k+\frac{\tilde{\alpha} k^{2}}{r_{+}^{2}}+\frac{r_{+}^{2}}{l^{2}}\right) .
$$

Thus, we have transformed the field equations in Guass-Bonnet gravity to the first law of thermodynamics, $T d S-d E=$ $P d V$, on the black hole horizon. The obtained expressions for the entropy and energy in (22) and (23), coincide with the expressions of entropy and energy for Gauss-Bonnet black holes in AdS spaces derived by solving the field equations [24].

In our analysis, we have supposed that both $l^{2}=-n(n-1) / \Lambda$ and $\tilde{\alpha}$ are fixed. Recently, there were a lot of interest in denoting $\Lambda$ as a thermodynamical variable proportional to the pressure 25]. In this case the last term in the left-hand side of Eq. (21), $n(n-1) /\left(16 \pi G_{n+1} l^{2}\right) d V$, may be moved to the right-hand side as $P_{\Lambda} d V$. One should note that the authors of Ref. 25] have considered the solutions of Einstein field equations, and therefore only the term $P_{\Lambda} d V$ will be appeared in the right-hand side of Eq. (21) for the solutions of Einstein equation. Also, one may note that $P_{\Lambda}$ is the $T_{r}^{r}$-component of energy momentum tensor of cosmological constant term, if one denotes the $\Lambda$-term as energy term in the right-hand side of Einstein equation. Also, if one denotes $\tilde{\alpha}$ as a thermodynamical variable, then a term $\tilde{\alpha} d A$ will appear in the right hand side of Eq. (21), where $A$ is the conjugate quantity to the Gauss-Bonnet coefficient [26]. In this case, since the Gauss-Bonnet coefficient has not the dimensions of pressure in geometric units, its conjugate quantity has not the dimensions of volume.

Here, before going to the case of quasi-topological gravity, we pause to give a comment on the expressions for energy density $\rho$ and pressure $P$. In general, these quantities are not the same for a general perfect fluid. Indeed $P$ is given in Eq. (19), while $\rho$ can be calculated by varying the action (10) with respect to $g_{t t}$ and multiplying both sides in $(-g)^{-1 / 2} g_{t t}$ as

$$
\frac{(n-1)}{16 \pi G_{n+1}} \frac{N}{r^{3}}\left\{\left(r^{2}+2 k \tilde{\alpha}-2 \tilde{\alpha} f\right) f^{\prime}+r(n-2)(f-k)-\frac{\tilde{\alpha}}{r}(n-4)\left[k^{2}-2 k f+f^{2}\right]-\frac{n r^{3}}{l^{2}}\right\}=-T_{t}^{t}=\rho .
$$

Of course, one may note that the pressure and energy density are the same on the horizon as one may see by calculating $\rho$ on the horizon.

\section{QUASI-TOPOLOGICAL GRAVITY}

The action of the quasi-topological theory in $(n+1)$-dimensions is given by

$$
I=\int d^{n+1} x\left(\mathcal{L}_{G}+\mathcal{L}_{M}\right),
$$


where $\mathcal{L}_{M}$ is the Lagrangian of the matter and

$$
\mathcal{L}_{G}=\frac{\sqrt{-g}}{16 \pi G_{n+1}}\left(-2 \Lambda+\mu_{1} \mathcal{L}_{1}+\mu_{2} \mathcal{L}_{2}+\mu_{3} \mathcal{X}_{3}+\mu_{4} \mathcal{X}_{4}+\ldots\right) .
$$

In the above equation $\Lambda=-(n-2)(n-3) / 2 l^{2}$ is the cosmological constant, $\mathcal{L}_{1}=R$ is the Einstein-Hilbert Lagrangian, $\mathcal{L}_{2}=R_{a b c d} R^{a b c d}-4 R_{a b} R^{a b}+R^{2}$ is the second order Lovelock (Gauss-Bonnet) Lagrangian, $\mathcal{X}_{3}$ is the curvature-cubed Lagrangian given by [16]

$$
\begin{aligned}
\mathcal{X}_{3}= & R_{a b}^{c d} R_{c d}^{e}{ }^{f} R_{e f}^{a b}+\frac{1}{(2 n-1)(n-3)}\left(\frac{3(3 n-5)}{8} R_{a b c d} R^{a b c d} R\right. \\
& -3(n-1) R_{a b c d} R^{a b c}{ }_{e} R^{d e}+3(n+1) R_{a b c d} R^{a c} R^{b d} \\
& \left.+6(n-1) R_{a}{ }^{b} R_{b}{ }^{c} R_{c}{ }^{a}-\frac{3(3 n-1)}{2} R_{a}^{b} R_{b}{ }^{a} R+\frac{3(n+1)}{8} R^{3}\right),
\end{aligned}
$$

and $\mathcal{X}_{4}$ is the fourth order term of quasi-topological gravity [17]

$$
\begin{aligned}
\mathcal{X}_{4}= & c_{1} R_{a b c d} R^{c d e f} R_{e f}^{h g} R_{h g}{ }^{a b}+c_{2} R_{a b c d} R^{a b c d} R_{e f} R^{e f}+c_{3} R R_{a b} R^{a c} R_{c}{ }^{b}+c_{4}\left(R_{a b c d} R^{a b c d}\right)^{2} \\
& +c_{5} R_{a b} R^{a c} R_{c d} R^{d b}+c_{6} R R_{a b c d} R^{a c} R^{d b}+c_{7} R_{a b c d} R^{a c} R^{b e} R_{e}^{d}+c_{8} R_{a b c d} R^{a c e f} R_{e}^{b} R_{f}^{d} \\
& +c_{9} R_{a b c d} R^{a c} R_{e f} R^{b e d f}+c_{10} R^{4}+c_{11} R^{2} R_{a b c d} R^{a b c d}+c_{12} R^{2} R_{a b} R^{a b} \\
& +c_{13} R_{a b c d} R^{a b e f} R_{e f}{ }_{g}^{c} R^{d g}+c_{14} R_{a b c d} R^{a e c f} R_{g e h f} R^{g b h d}
\end{aligned}
$$

where the coefficients $c_{i}$ are given by [17]

$$
\begin{aligned}
c_{1} & =-(n-1)\left(n^{7}-3 n^{6}-29 n^{5}+170 n^{4}-349 n^{3}+348 n^{2}-180 n+36\right), \\
c_{2} & =-4(n-3)\left(2 n^{6}-20 n^{5}+65 n^{4}-81 n^{3}+13 n^{2}+45 n-18\right), \\
c_{3} & =-64(n-1)\left(3 n^{2}-8 n+3\right)\left(n^{2}-3 n+3\right), \\
c_{4} & =-\left(n^{8}-6 n^{7}+12 n^{6}-22 n^{5}+114 n^{4}-345 n^{3}+468 n^{2}-270 n+54\right), \\
c_{5} & =16(n-1)\left(10 n^{4}-51 n^{3}+93 n^{2}-72 n+18\right), \\
c_{6} & =--32(n-1)^{2}(n-3)^{2}\left(3 n^{2}-8 n+3\right), \\
c_{7} & =64(n-2)(n-1)^{2}\left(4 n^{3}-18 n^{2}+27 n-9\right), \\
c_{8} & =-96(n-1)(n-2)\left(2 n^{4}-7 n^{3}+4 n^{2}+6 n-3\right), \\
c_{9} & =16(n-1)^{3}\left(2 n^{4}-26 n^{3}+93 n^{2}-117 n+36\right), \\
c_{10} & =n^{5}-31 n^{4}+168 n^{3}-360 n^{2}+330 n-90, \\
c_{11} & =2\left(6 n^{6}-67 n^{5}+311 n^{4}-742 n^{3}+936 n^{2}-576 n+126\right), \\
c_{12} & =8\left(7 n^{5}-47 n^{4}+121 n^{3}-141 n^{2}+63 n-9\right), \\
c_{13} & =16 n(n-1)(n-2)(n-3)\left(3 n^{2}-8 n+3\right), \\
c_{14} & =8(n-1)\left(n^{7}-4 n^{6}-15 n^{5}+122 n^{4}-287 n^{3}+297 n^{2}-126 n+18\right) .
\end{aligned}
$$

For spherically symmetric metric, the action (25) yields second-order equations of motion in $(n+1)$-dimensional spacetimes except in $n=2 m-1$, where $m$ is the order of quasi-topological theory [17]. In the remaining part of this paper, we will show that the gravitational field equations describing by the spacetime metric (18) can be recast in the form of the first law of thermodynamics at the black hole horizon.

\section{HORIZON THERMODYNAMICS IN CUBIC QUASI-TOPOLOGICAL GRAVITY}

In section II, we showed that the field equations of static black hole spacetimes, with constant horizon curvature, in Einstein and Gauss-Bonnet gravities can be reexpressed as the first law of thermodynamics, $d E=T d S-P d V$, on the horizon. Here, we want to see whether the above procedure works or not in other gravity theories such as quasi-topological gravity. As we discussed, and explicitly showed, for the Gauss-Bonnet case, one can use both the field equations as well as the variational principle, instead of using the field equations, and transform the equations 
of motion to the first law at the spacetime horizon. However, since the field equations for cubic [16] and quartic [27] quasi-topological gravity are very long, in this section and also the next one, we present the resulting equations from variational principle for economic reason, which are clearly the components of the field equations.

We shall now continue the previous procedure for the cubic term of quasi-topological gravity. The total action of the cubic quasi-topological gravity in $(n+1)$ dimensions can be written as

$$
I=\frac{1}{16 \pi G_{n+1}} \int d^{n+1} x \sqrt{-g}\left[-2 \Lambda+\mu_{1} \mathcal{L}_{1}+\mu_{2} \mathcal{L}_{2}+\mu_{3} \mathcal{X}_{3}\right]+\int d^{n+1} x \mathcal{L}_{M}
$$

Varying the action (29) with respect to $g^{r r}$ and multiplying both sides in $(-g)^{-1 / 2} g^{r r}$, one obtains

$$
\begin{aligned}
& \frac{(n-1)}{16 \pi G_{n+1}} \frac{1}{N r^{5}}\left\{\frac{d}{d r}\left(N^{2} f\right)\left(r^{4}+2 k r^{2} \hat{\mu}_{2} l^{2}-2 r^{2} \hat{\mu}_{2} l^{2} f+3 k^{2} \hat{\mu}_{3} l^{4}-6 k \hat{\mu}_{3} l^{4} f+3 k \hat{\mu}_{3} l^{4} f^{2}\right)\right. \\
& \left.+N^{2}\left[r^{3}(n-2)(f-k)-\hat{\mu}_{2} l^{2} r(n-4)\left(k^{2}-2 k f+f^{2}\right)-\frac{\hat{\mu}_{3}}{r} l^{4}(n-6)\left(-k+3 k^{2} f-3 k f^{2}+f^{3}\right)-\frac{n r^{5}}{l^{2}}\right]\right\}=T_{r}^{r},
\end{aligned}
$$

where $T_{r}^{r}$ is given by (17), and

$$
\hat{\mu}_{1}=1, \quad \hat{\mu}_{2}=\frac{(n-2)(n-3)}{l^{2}} \mu_{2}, \quad \hat{\mu}_{3}=\frac{(n-2)(n-5)\left(3 n^{2}-9 n+4\right)}{8(2 n-1) l^{4}} \mu_{3} .
$$

Next, we evaluate Eq. (30) at $r=r_{+}$and using the fact that $f\left(r_{+}\right)=0$, to obtain

$$
\begin{aligned}
& \frac{N(n-1)}{16 \pi G_{n+1}}\left\{f^{\prime}\left(r_{+}\right)\left(r_{+}+\frac{2 k}{r_{+}} \hat{\mu}_{2} l^{2}+\frac{3 k^{2}}{r_{+}^{3}} \hat{\mu}_{3} l^{4}\right)-k(n-2)\right. \\
& \left.-\frac{\hat{\mu}_{2} l^{2}}{r_{+}^{2}} k^{2}(n-4)-\frac{\hat{\mu}_{3} l^{4} k}{r_{+}^{4}}(n-6)-\frac{n r_{+}^{2}}{l^{2}}\right\}=r_{+}^{2} P .
\end{aligned}
$$

Multiplying both sides of the above equation by the factor $\Sigma_{k} r_{+}^{n-3} d r_{+}$, and setting $f^{\prime}\left(r_{+}\right)=2 \kappa$, we have

$$
\begin{aligned}
& \frac{\kappa}{2 \pi} d\left[\frac{\Sigma_{k} r_{+}^{n-1}}{4 G_{n+1}}\left(1+2 k \hat{\mu}_{2} \frac{(n-1)}{(n-3)} \frac{l^{2}}{r_{+}^{2}}+3 k^{2} \hat{\mu}_{3} \frac{(n-1)}{(n-5)} \frac{l^{4}}{r_{+}^{4}}\right)\right] \\
& -d\left[\frac{(n-1) \Sigma_{k} r_{+}^{n-2}}{16 \pi G_{n+1}}\left(k+\frac{k^{2} \hat{\mu}_{2} l^{2}}{r_{+}^{2}}+\frac{k^{3} \hat{\mu}_{3} l^{4}}{r_{+}^{4}}+\frac{r_{+}^{2}}{l^{2}}\right)\right]=P \Sigma_{k} r+{ }^{n-1} d r_{+} .
\end{aligned}
$$

We can rewrite this equation in the form,

$$
\begin{aligned}
& T d\left[\frac{\Sigma_{k} r_{+}^{n-1}}{4 G_{n+1}}\left(1+2 k \hat{\mu}_{2} \frac{(n-1)}{(n-3)} \frac{l^{2}}{r_{+}^{2}}+3 k^{2} \hat{\mu}_{3} \frac{(n-1)}{(n-5)} \frac{l^{4}}{r_{+}^{4}}\right)\right] \\
& -d\left[\frac{(n-1) \Sigma_{k} r_{+}^{n}}{16 \pi G_{n+1} l^{2}}\left(1+k \frac{l^{2}}{r_{+}^{2}}+k^{2} \hat{\mu}_{2} \frac{l^{4}}{r_{+}^{4}}+k^{3} \hat{\mu}_{3} \frac{l^{6}}{r_{+}^{6}}\right)\right]=P d V .
\end{aligned}
$$

The first term in the left hand side of the above equation is in the form $T d S$, and so one may recognize the entropy expression for the horizon in cubic quasi-topological gravity as,

$$
S=\frac{\Sigma_{k} r_{+}^{n-1}}{4 G_{n+1}}\left(1+2 k \hat{\mu}_{2} \frac{(n-1)}{(n-3)} \frac{l^{2}}{r_{+}^{2}}+3 k^{2} \hat{\mu}_{3} \frac{(n-1)}{(n-5)} \frac{l^{4}}{r_{+}^{4}}\right) .
$$

According to the first low of thermodynamics, we can interpret the second term in the left hand side of (33) as the energy of the system,

$$
E=\frac{(n-1) \Sigma_{k} r_{+}^{n}}{16 \pi G_{n+1} l^{2}}\left(1+k \frac{l^{2}}{r_{+}^{2}}+k^{2} \hat{\mu}_{2} \frac{l^{4}}{r_{+}^{4}}+k^{3} \hat{\mu}_{3} \frac{l^{6}}{r_{+}^{6}}\right)
$$


These expressions for entropy and energy of the black holes coincide exactly with those obtained in quasi-topological gravity by solving the field equations and using the Wald's method [16]. Here we arrived at the same result by transforming the field equations to the form of the first law on the black hole horizon. This indicates that the approach presented here is enough powerful and further reveals the deep connection between the gravitational field equations and the first law of thermodynamics on the horizon of the black hole. Again, one can show that although $P$ and $\rho$ are different in general but they are the same at the horizon.

\section{HORIZON THERMODYNAMICS OF QUASI-TOPOLOGICAL GRAVITY}

In this section, we would like to extend the above study to the case of $m$-th order quasi-topological gravity. First, we consider the quartic quasi-topological gravity. Varying the action (25) with respect to $g^{r r}$ and multiplying both sides in $(-g)^{-1 / 2} g^{r r}$, we obtain

$$
\begin{aligned}
& \frac{(n-1)}{16 \pi G_{n+1}} \frac{1}{N r^{7}}\left\{\frac{d}{d r}\left(N^{2} f\right)\left(r^{6}+2 k r^{4} \hat{\mu}_{2} l^{2}-2 r^{4} \hat{\mu}_{2} l^{2} f+3 k^{2} r^{2} \hat{\mu}_{3} l^{4}-6 k r^{2} \hat{\mu}_{3} l^{4} f+3 r^{2} \hat{\mu}_{3} l^{4} f^{2}\right)\right. \\
& +N^{2}\left[f^{\prime}\left(4 k \hat{\mu}_{4} l^{6}-12 k^{2} \hat{\mu}_{4} l^{6} f+12 k \hat{\mu}_{4} l^{6} f^{2}-4 \hat{\mu}_{4} l^{6} f^{3}\right)+r^{5}(n-2)(f-k)+\hat{\mu}_{2} l^{2} r^{3}(n-4)\left(2 k f-k^{2}-f^{2}\right)\right. \\
& \left.\left.-\hat{\mu}_{3} l^{4} r(n-6)\left(k-3 k^{2} f+3 k f^{2}-f^{3}\right)+\frac{\hat{\mu}_{4}}{r} l^{6}(n-8)\left(-k^{2}+4 k f-6 k^{2} f^{2}+4 k f^{3}-f^{4}\right)-\frac{n r^{7}}{l^{2}}\right]\right\}=T_{r}^{r},
\end{aligned}
$$

where

$$
\hat{\mu}_{4}=\frac{n(n-1)(n-2)^{2}(n-3)(n-7)\left(n^{5}-15 n^{4}+72 n^{3}-156 n^{2}+150 n-42\right)}{l^{6}} \mu_{4} .
$$

Evaluating Eq. (36) at $r=r_{+}$and setting $f\left(r_{+}\right)=0$, we arrive at

$$
\begin{aligned}
& \frac{N(n-1)}{16 \pi G_{n+1}}\left\{f^{\prime}\left(r_{+}+\frac{2 k}{r_{+}} \hat{\mu}_{2} l^{2}+\frac{3 k^{2}}{r_{+}^{3}} \hat{\mu}_{3} l^{4}+\frac{4 k}{r_{+}^{5}} \hat{\mu}_{4} l^{6}\right)-k(n-2)\right. \\
& \left.-\hat{\mu}_{2} \frac{k^{2} l^{2}}{r_{+}^{2}}(n-4)-\hat{\mu}_{3} \frac{k l^{4}}{r_{+}^{4}}(n-6)-\frac{\hat{\mu}_{4} l^{6} k^{2}}{r_{+}^{6}}(n-8)-\frac{n r_{+}^{2}}{l^{2}}\right\}=P r_{+}^{2},
\end{aligned}
$$

Multiplying both sides of the above equation by the factor $\Sigma_{k} r_{+}^{n-3} d r_{+}$and setting $f^{\prime}\left(r_{+}\right)=2 \kappa$, we get

$$
\begin{aligned}
& \frac{\kappa}{2 \pi} d\left[\frac{\Sigma_{k} r_{+}^{n-1}}{4 G_{n+1}}\left(1+2 k \hat{\mu}_{2} \frac{(n-1)}{(n-3)} \frac{l^{2}}{r_{+}^{2}}+3 k^{2} \hat{\mu}_{3} \frac{(n-1)}{(n-5)} \frac{l^{4}}{r_{+}^{4}}+4 k^{3} \hat{\mu}_{4} \frac{(n-1)}{(n-7)} \frac{l^{6}}{r_{+}^{6}}\right)\right] \\
& -d\left[\frac{(n-1) \Sigma_{k} r_{+}^{n-2}}{16 \pi G_{n+1}}\left(k+\frac{\hat{\mu}_{2} l^{2} k^{2}}{r_{+}^{2}}+\frac{\hat{\mu}_{3} l^{4} k^{3}}{r_{+}^{4}}+\frac{\hat{\mu}_{4} l^{6} k^{4}}{r_{+}^{6}}+\frac{r_{+}^{2}}{l^{2}}\right)\right]=P \Sigma_{k} r+{ }^{n-1} d r_{+}
\end{aligned}
$$

Using the definition $T=\kappa / 2 \pi$, the above equation can be rewritten in the form

$$
\begin{aligned}
& T d\left[\frac{\Sigma_{k} r_{+}^{n-1}}{4 G_{n+1}}\left(1+2 k \hat{\mu}_{2} \frac{(n-1)}{(n-3)} \frac{l^{2}}{r_{+}^{2}}+3 k^{2} \hat{\mu}_{3} \frac{(n-1)}{(n-5)} \frac{l^{4}}{r_{+}^{4}}+4 k^{3} \hat{\mu}_{4} \frac{(n-1)}{(n-7)} \frac{l^{6}}{r_{+}^{6}}\right)\right] \\
& -d\left[\frac{(n-1) \Sigma_{k} r_{+}^{n}}{16 \pi G_{n+1} l^{2}}\left(1+k \frac{l^{2}}{r_{+}^{2}}+\hat{\mu}_{2} k^{2} \frac{l^{4}}{r_{+}^{4}}+\hat{\mu}_{3} k^{3} \frac{l^{6}}{r_{+}^{6}}+\hat{\mu}_{4} k^{4} \frac{l^{8}}{r_{+}^{8}}\right)\right]=P d V .
\end{aligned}
$$

Equation (39) is nothing, but the first law of thermodynamics on the horizon, $T d S-d E=P d V$. We can define the entropy expression as

$$
S=\frac{\Sigma_{k} r_{+}^{n-1}}{4 G_{n+1}}\left(1+2 k \hat{\mu}_{2} \frac{(n-1)}{(n-3)} \frac{l^{2}}{r_{+}^{2}}+3 k^{2} \hat{\mu}_{3} \frac{(n-1)}{(n-5)} \frac{l^{4}}{r_{+}^{4}}+4 k^{3} \hat{\mu}_{4} \frac{(n-1)}{(n-7)} \frac{l^{6}}{r_{+}^{6}}\right),
$$

and the total energy (mass) of the black hole as

$$
E=\frac{(n-1) \Sigma_{k} r_{+}^{n}}{16 \pi G_{n+1} l^{2}}\left(1+k \frac{l^{2}}{r_{+}^{2}}+\hat{\mu}_{2} k^{2} \frac{l^{4}}{r_{+}^{4}}+\hat{\mu}_{3} k^{3} \frac{l^{6}}{r_{+}^{6}}+\hat{\mu}_{4} k^{4} \frac{l^{8}}{r_{+}^{8}}\right) .
$$


The obtained expressions for entropy and energy are precisely the expressions calculated by other authors for topological black holes in quartic quasi-topological gravity [17]. In this way we show that the field equations of quartic quasi-topological gravity can be transformed to the form of the first law of thermodynamics on the event horizon of $(n+1)$-dimensional topological black holes.

Having the results for the cubic and quartic cases at hand, one may conjecture that there exists similar connection for $m$-th order quasi-topological gravity in $n \neq 2 m-1$ dimensions. For the topological black holes with metric (18), one may conjecture that the gravity part of the action (25) is given by [17]

$$
I_{G}=\int d t d r N(r)\left(r^{n} \sum_{i=0}^{m} \hat{\mu}_{i}\left[l^{2} r^{-2}(k-f)\right]^{i}\right)^{\prime},
$$

where $\hat{\mu}_{i}$ are coefficients of the $i$-th powered curvature term with $\hat{\mu}_{1}=1$. The corresponding field equations evaluated on the horizon may be rewritten as

$$
\operatorname{Td}\left[\frac{\Sigma_{k} r_{+}^{n-1}}{4 G_{n+1}} \sum_{i=1}^{m} i \frac{(n-1)}{(n+1-2 i)} \frac{\hat{\mu}_{i} k^{i-1} l^{2 i-2}}{r_{+}^{2 i-2}}\right]-d\left[\frac{(n-1) \Sigma_{k} r_{+}^{n}}{16 \pi G_{n+1} l^{2}}\left(1+\sum_{i=1}^{m} \hat{\mu}_{i} k^{i} \frac{l^{2 i}}{r_{+}^{2 i}}\right)\right]=P d V .
$$

Equation (43) is the first law of thermodynamics provided we define

$$
\begin{aligned}
& S=\frac{\Sigma_{k} r_{+}^{n-1}}{4 G_{n+1}} \sum_{i=1}^{m} i \frac{(n-1)}{(n+1-2 i)} \frac{\hat{\mu}_{i} k^{i-1} l^{2 i-2}}{r_{+}^{2 i-2}}, \\
& E=\frac{(n-1) \Sigma_{k} r_{+}^{n}}{16 \pi G_{n+1} l^{2}}\left(1+\sum_{i=1}^{m} \hat{\mu}_{i} k^{i} \frac{l^{2 i}}{r_{+}^{2 i}}\right) .
\end{aligned}
$$

These are the most general expressions for entropy and energy of static black hole spacetimes with spherical, hyperbolic or planar horizon topology in the most general quasi-topological theory of gravity. We expect to confirm our general results (44) and (44) in the future by solving explicitly the field equations. Again, one can show that although $P$ and $\rho$ are different in general but they are the same at the horizon.

\section{CONCLUSIONS}

According to the black hole thermodynamics, a black hole can be regarded as a thermodynamic system which has entropy and temperature associated with its horizon. Since the discovery of black hole thermodynamics in 1970's physicists have been speculating that there should be some deep connection between thermodynamics and gravity. This is due to the fact that thermodynamic quantities of black holes such as temperature and entropy are closely related to their geometrical quantities such as surface gravity and horizon area. In this paper, we have investigated the thermodynamics of topological black holes, with spherical, hyperbolic or planar horizon topology, in quasi-topological theory of gravity. We showed that one can always rewrite the field equations of quasi-topological gravity in the form of the first law of thermodynamics, $d E=T d S-P d V$, at the black hole horizon. This procedure allows us to obtain the entropy and the mass expressions in terms of the radius of black hole horizon, which are exactly the same as those resulting from the Wald's method for black hole entropy and the Hamiltonian approach for black hole mass. The novelty and advantages of the present study is that in the process of deriving the entropy and the mass of black holes, we have not solved the field equation of the quasi-topological theory and we had no difficulties of Wald's method for calculating entropy. This completely differs from the previous works in the literature [16, 17].

The thermodynamic interpretation of the gravitational field equations in the most general quasi-topological gravity indicates that the connection between thermodynamics and gravity is not just an accident, but something with deep physical meaning. On the other hand the disclosed relation on the field equations and the first law of thermodynamics on the black hole horizon also sheds the light on holography, since the gravitational field equations persists the information in the bulk and the first law of thermodynamics on the event horizon contains the information on the boundary. Our study shows that the approach here is powerful to find an expression of entropy in term of the horizon radius. It could help to extract an expression of entropy associated with the event horizon in quasi-topological gravity, which is useful in studying the thermodynamical properties of black holes in this theory. 


\section{Acknowledgments}

We thank from the Research Council of Shiraz University. This work has been supported financially by Research Institute for Astronomy \& Astrophysics of Maragha (RIAAM), Iran.

[1] T. Jacobson, Phys. Rev. Lett. 75, 1260 (1995).

[2] R. G. Cai and S. P. Kim, J. High Energy Phys 0502, 050 (2005).

[3] A. Paranjape, S. Sarkar and T. Padmanabhan, Phys. Rev. D 74, 104015 (2006).

[4] T. Padmanabhan, Rept. Prog. Phys. 73 (2010) 046901;

T. Padmanabhan, Mod. Phys. Lett. A 25 (2010) 1129;

T. Padmanabhan, Phys. Rev. D 81, 124040 (2010).

[5] M. Akbar and R. G. Cai, Phys. Lett. B 648, 243 (2007).

[6] M. Akbar and R. G. Cai, Phys. Lett. B 635, 7 (2006).

[7] M. Akbar and R. G. Cai, Phys. Rev. D 75, 084003 (2007).

[8] R. G. Cai and L. M. Cao, Phys.Rev. D 75, 064008 (2007).

[9] R. G. Cai and L. M. Cao, Nucl. Phys. B 785, 135 (2007).

[10] A. Sheykhi, B. Wang and R. G. Cai, Nucl. Phys. B 779, 1 (2007).

[11] A. Sheykhi, B. Wang and R. G. Cai, Phys. Rev. D 76, 023515 (2007);

A. Sheykhi, J. Cosmol. Astropart. Phys. 05, 019 (2009).

[12] A. Sheykhi, Eur. Phys. J. C 69, 265 (2010);

A. Sheykhi, Class. Quantum Gravit. 27, (2010) 025007;

A. Sheykhi, Phys. Rev. D 87, 024022 (2013).

[13] R. G. Cai and N. Ohta, Phys. Rev. D 81, (2010) 084061.

[14] D. Lovelock, J. Math. Phys. (N.Y.) 12, 498 (1971).

[15] J. Oliva and S. Ray, Classical Quantum Gravity 27, 225002 (2010).

[16] R. C. Myers and B. Robinson, J. High Energy Phys. 08067 (2010).

[17] M. H. Dehghani, et. al., Phys. Rev. D 85, 104009 (2012).

[18] R. C. Myers, M. F. Paulos and A. Sinha, Phys. Rev. D 79, 041901 (2009);

A. Buchel, R. C. Myers, M. F. Paulos and A. Sinha, Phys. Lett. B 669, 364 (2008);

A. Buchel, R. C. Myers and A. Sinha, J. High Energy Phys. 03 (2009) 084;

A. Sinha and R. C. Myers, Nucl. Phys. A 830, 295C (2009);

R. C. Myers, M. F. Paulos, and A. Sinha, J. High Energy Phys. 08035 (2010).

[19] W. G. Brenna, M. H. Dehghani, and R. B. Mann, Phys. Rev. D 84, 024012 (2011);

M.H. Dehghani and M. H. Vahidinia, Phys. Rev. D 84, 084044 (2011).

[20] W. G. Brenna and R. B. Mann, Phys. Rev. D 86, 064035 (2012) .

[21] A. Bazrafshan, M. H. Dehghani, M. Ghanaatian, Phys. Rev. D 86, 104043 (2012).

[22] Xiao-Mei Kuang, Wei-Jia Li, Yi Ling, J. High Energy Phys 1012, 069 (2010); Xiao-Mei Kuang, Wei-Jia Li, Yi Ling, Class. Quantum Grav. 29, 085015 (2012).

[23] B. Zwiebach, Phys. Lett. 156B, 315 (1985);

B. Zumino, Phys. Rept. 137, 109 (1986);

D.J. Gross and J.H. Sloan, Nucl. Phys. B 291, 41 (1987).

[24] R. G. Cai, Phys. Rev. D 65, 084014 (2002);

R. G. Cai and Q. Guo, Phys. Rev. D 69, 104025 (2004);

R. G. Cai and K. S. Soh, Phys. Rev. D 59, 044013 (1999).

[25] D. Kastor, S. Ray and J. Traschen, Class. Quant. Grav. 26, 195011 (2009);

B. Dolan, Class. Quant. Grav. 28, 125020 (2011);

B. P. Dolan, Class. Quant. Grav. 28, 235017 (2011);

B. P. Dolan, Phys. Rev. D 84, 127503 (2011);

M. Cvetic, G. Gibbons, D. Kubiznak, and C. Pope, Phys. Rev. D 84, 024037 (2011);

D. Kubiznak and R. B. Mann, J. High Energy Phys. 07, 033 (2012);

S. Gunasekaran, R. B. Mann and D. Kubiznak, J. High Energy Phys. 11, 110 (2012)

B. P. Dolan, D. Kastor, D. Kubiznak, R. B. Mann and J. Traschen, Phys. Rev. D 87, 104017 (2013);

S. H. Hendi and M. H. Vahidinia, Phys. Rev. D 88, 084045 (2013).

[26] R. G. Cai, L. M. Cao, L. Li and R. Q. Yang, J. High Energy Phys. 09, 005 (2013).

[27] M. H. Dehghani and M. H. Vahidinia, J. High Energy Phys. 10, 210 (2013). 